\title{
Originalia
}

\section{Unterstützung von Spiritual Care durch räumliche Gestaltung von Gesundheitsbauten}

\author{
Health Design in Support of Spiritual Care
}

https://doi.org/10.1515/spircare-2018-0041

Zusammenfassung: Die räumliche Gestaltung von Gesundheitsbauten kann spirituelle Bedürfnisse und Spiritual Care behindern oder begünstigen. Patienten, Besucher und Personal erleben beispielsweise den Charakter eines Krankenzimmers als oft durchaus eindringliche Anmutungen und Stimmungen, sogenannte Atmosphären. Solches Erleben ist leiblich-räumlich. Ebenso ist es vieldimensional synästhetisch, integriert alle Sinne sowie Sinne und Geist. ${ }^{1}$ Der ganze Mensch ist davon betroffen und berührt, wie die Umgebung gestaltet ist, als Patient oft auf besonders intensive Weise. Doch wie lassen sich Räume so gestalten, dass ihre Grundstimmung spirituelle Bedürfnisse und Spiritual Care unterstützt? Inspiration läßt sich aus Erkenntnissen zum Welt-Erleben von Patienten, aus Ansätzen der „Healing Architecture“ und aus der Gestaltung sakraler Bauten und Räume gewinnen.

Schlüsselwörter: Atmosphären-Gestaltung, räumliche Umsetzung spiritueller Bedürfnisse, leiblich-synästhetisches Erleben

Summary: Healthcare design may significantly contribute to atmospheres that are supportive of spiritual needs. Fundamental for the perception and thus design of spiritual spaces is the dimension of bodily synaesthetic experience. Healthcare design in the spirit of spiritual care should be informed by knowledge about relevant patient perceptions such as the disturbed and distorted character of proprioceptive perception in some cancer patients.

1 Der Ausdruck „Synästhesie“ wird hier bewusst liberal in einer informellen und tendenziell weiten Bedeutung verstanden. Diese entspricht einer in vielen Kontexten etablierten und nach meinem Dafürhalten sachdienlichen Verwendung, auch wenn die originale sinnesphysiologische Definition dabei gelockert, erweitert und teilweise umgedeutet erscheint.

*Korrespondenzautorin: Katharina Brichetti, Berlin,

E-Mail: studio-kb@gmx.de
Keywords: design of atmospheres, spiritual spaces, bodily synaesthetic experience

Heilung im umfassenden Sinne ist weit mehr als ein biologischer Prozess, da sie den ganzen Menschen betrifft. Neben der körperlichen Gesundung gehören zur Genesung psychische, soziale und auch spirituelle Aspekte. Immer noch behandeln Mediziner den Kranken im Wesentlichen als zu reparierende biologische Maschine. Doch kommt in jüngerer Zeit zunehmend in den Blick, wie stark Heilung auch das Erleben des Leidenden, seine Gedanken und Empfindungen betrifft und von diesen abhängt, im Einklang mit der WHO-Definition von Gesundheit als „Zustand des vollständigen körperlichen, sozialen und geistigen Wohlergehens, nicht nur des Fehlens von Krankheit oder Gebrechen“ (Verfassung der WHO).

In den letzten Jahren wächst das Bewusstsein, wie entscheidend auch die räumliche Gestaltung von Krankenzimmern und Krankenhaus dazu beiträgt, ob Patienten sich in ihren emotionalen und seelischen Bedürfnissen geachtet und unterstützt fühlen. Unter Schlagworten wie „Healing Architecture“ und „Healing Design“ formiert sich eine Bewegung, welche die übliche steril-funktionale Gestaltung von Gesundheitsbauten ablehnt und Räume zum Wohlfühlen fordert, etwa ausgedrückt in der Forderung nach Krankenhäusern mit der Atmosphäre eines guten Hotels oder Wellness-Zentrums. Angesichts dieser wichtigen und erfreulichen Entwicklungen sollte allerdings nicht vergessen werden: Zum menschlichen Wohlergehen gehört mehr als Wohlfühlen im Sinne von Wellness. Gerade in der Krankheit können seelische Bedürfnisse existenziell werden, die ihre Erfüllung keineswegs in Komfort und erlebter Wohligkeit finden: etwa die Suche nach dem Sinn des Leidens, des eigenen Lebens und des Ganzen, die Sehnsucht nach seelisch-geistiger Erfüllung und innerem Wachstum, der tiefe Wunsch, sich mit der Situation und der Endlichkeit des Lebens zu versöhnen, mehr Weisheit, Achtsamkeit, Mitgefühl, Liebesfähigkeit zu entwickeln, das Ego loslassen zu können. „Spiritual Care“ nimmt diese 
im weitesten Sinne spirituellen Bedürfnisse und Sehnsüchte ernst als für den Menschen und gerade für den Kranken bedeutsame Dimension des Lebens. „Spiritual Care" kann noch seelisch heilend wirken, wo die Hoffnung auf körperliche Heilung aufgegeben werden muss. Können Architektur und Design zur spirituellen Dimension der Heilung und zum spirituellen Wohlergehen beitragen? Lassen sich spirituelle Bedürfnisse und Spiritual Care gestalterisch unterstützen - auch über die bloße Wohlfühlatmosphäre hinaus? Bei Projekten mit dem Anspruch von „Healing Architekture“ oder „Healing Design“ geht es oft zunächst und basal darum, den Menschen etwas zu bieten, wie etwa die besagte Atmosphäre eines Hotels oder Wellness-Zentrums. Spirituelle Bedürfnisse sind nun aber gerade keine Konsum-Bedürfnisse. Die spirituellen Dimensionen des Lebens bedeuten wesentlich Sorge um und für sich selbst, Sorge um und für den Mitmenschen und das Leben insgesamt, sie sind aktive seelische Entwicklung und innerer Einsatz. Sie beruhen auf Haltung und Einstellung, auf einem inneren Appell, auf der Entschlossenheit, auf diesem Weg der Seele fortzuschreiten. So lassen sich eine schöne Umgebung, menschliche Liebe und Zuwendung einerseits passiv als Beiträge zum Wohlbefinden konsumieren, andererseits aber auch aktiv, als Anlass zur Dankbarkeit und damit als spiritueller Appell erleben.

Gespräche, Rituale, das Erleben von Schönheit, $\mathrm{Zu}$ wendung und Liebe können hier hilfreich beitragen und den kranken Menschen unterstützen. Doch Architektur und Design? Zunächst: Eine aufmerksam und liebevoll gestaltete Umgebung wird als solche auch wahrgenommen. Sie kann beispielsweise mit Dankbarkeit und dem Empfinden, in einem Größeren aufgehoben und geborgen zu sein - also spirituell - beantwortet werden. Sodann: Die menschliche Überzeugung, dass Architektur und Design Spiritualität anregen und unterstützen können, manifestiert sich seit Tausenden von Jahren über die ganze Welt hin in äußerlich und innerlich eindrucksvollen und berührenden Bauten sakraler Architektur, in Andachtsplätzen, Kirchen, Tempeln und Klöstern. Wäre es nicht nahe liegend, hier nach Anregungen zu suchen, wie Räume sich so gestalten lassen, dass sie spirituelle Bedürfnisse und Spiritual Care unterstützen? Es ist eine zentrale These dieses Beitrag, dass dies möglich ist.

Es ist Allgemeingut, dass Heilung verschiedene Aspekte hat und auf verschiedenen Ebenen stattfindet. Es ist dabei nicht erstaunlich, dass der Begriff der Heilung nicht eindeutig definiert ist. Je nach Fachgebieten gibt es bei der Definition von Heilung Unterschiede: So wird die Wiederherstellung der psychischen Gesundheit in der Psychotherapie mit dem Heilungsprozess gleichgestellt. Selbst innerhalb eines Faches können sich Ärzte in ihren Vorstel- lungen unterscheiden, was Gesundheit und Heilung bedeuten.

\section{Spiritualität fördert körperliche, geistige und seelische Heilung}

Auch die spirituelle Ebene kann für den Prozess der Heilung entscheidend sein und ist eine wesentliche und zeitlose Dimension des Menschseins. Spiritualität kann Lebensqualität und Wohlbefinden verbessern, weshalb sie im onkologischen Kontext berücksichtigt werden sollte (Bruns et al. 2007). Zum Beispiel können Menschen mit einer chronischen Krankheit lernen, in Frieden trotz ihres Zustandes zu sein. So umfasst die ganzheitliche Heilung neben der physischen Genesung auch psychische und spirituelle Gesichtspunkte. Vor allem im Bereich der Palliativmedizin hat sich zunehmend die Erkenntnis durchgesetzt, dass der Bedarf an Spiritualität und Fürsorge eine vierte wichtige Dimension neben der medizinischen, sozialen und psychologischen Dimension der angemessenen Sorge für den Kranken und des Kranken für sich selbst darstellt.

\section{Spiritual Care}

Seit den 1990er Jahren deckt sich der Begriff der Spiritualität ,längst nicht mehr mit Religiosität“, ist aber auch nicht von ihr zu trennen (Frick 2007: 12). Dementsprechend offen ist Traugott Rosers Definition von Spiritual Care. Sie sei „die Sorge um die individuelle Teilnahme und Teilhabe an einem als sinnvoll erfahrenen Leben in einem umfassenden Verständnis“ (Roser 2009: 88). Längst hat sich „die Bindung an Kirchen und andere religiöse Institutionen " in unserer pluralistischen, multikulturellen und globalisierten Welt gelockert (Grom 2011: 12). Spiritualität ist ein zeitgemäßer Begriff, der sich an der „subjektiven Erfahrung und Intuition“ des einzeln orientiert (Grom 2011: 13).

Eckhard Frick und Traugott Roser waren seit 2010 europaweit die ersten Professoren für die neue Disziplin Spiritual Care an der Ludwig-Maximilians-Universität (LMU) München. Aufgabe dieses neuen Fachgebietes war es, die spirituellen Bedürfnisse von schwerstkranken Patienten und ihren Familien, aber beispielsweise auch der sie betreuenden Ärzte und Pflegekräfte zu thematisieren. Nach Fricks These hat Spiritualität nichts mehr mit kirchlicher Trägerschaft von Krankenhäusern oder mit Krankenhausseelsorge im engeren Sinn zu tun, jedenfalls nicht in erster Linie. Vielmehr geht es Eckhard Frick um die „Gren- 
ze des Wiss- und Machbaren, an die Ärzte, Pflegepersonal und Patienten immer wieder geraten“ (Frick 2007: 27). Im Angesicht einer schweren Krankheit und des bevorstehenden Todes stellen sich neue Sinnfragen. Was ist im Angesicht des Todes das wirklich Wesentliche? Was bleibt am Ende des Lebens übrig? Was ist unser Sinn oder was ist unsere Ausrichtung? Was heißt es, bezüglich des eigenen Lebens über die eigene Endlichkeit hinauszublicken? „In einem erweiterten Sinn kann Heilung nämlich auch dann noch geschehen, wenn die Mittel der ärztlichen Kunst ausgeschöpft sind und es in erster Linie um die Versöhnung mit der Begrenztheit des endlichen Lebens (healing) geht" (Frick 2007: 20). Heil werden versteht man nicht „im Sinn einer idealen Stärke, Schönheit und Gesundheit, sondern im Sinn des Annehmens von Begrenzungen und auch bleibenden Wunden“ (Frick 2007: 74). „Zwar ist die Wiederherstellung der Gesundheit (to cure) nicht mehr möglich und der Tod absehbar, wohl aber bleibt die seelische und spirituelle Heilung (to heal) bis zum Lebensende möglich“ (Frick 2007: 76).

\section{Kerndimensionen spiritueller Bedürfnisse}

„Spiritualität gilt in den Gesundheitswissenschaften als der umfassende Begriff. Spiritual Care benötigen nicht nur religiöse Menschen. Auch Patienten, die einen religiösen Glauben oder die Zugehörigkeit $\mathrm{zu}$ einer religiösen Gemeinschaft verneinen, stellen sich die Frage nach dem ,Warum““, nach dem Sinn von Krankheit und Sterben. Sie haben das Recht auf unsere Begleitung, auch dann, wenn sie nicht mit dem Seelsorger sprechen möchten. Deshalb muss Spiritual Care interreligiös und überkonfessionell offen sein, um der Vielfalt spiritueller Orientierungen bei Patienten, aber auch bei den Gesundheitsberufen zu entsprechen" (Boothe \& Frick 2017: 142). Dieser weit gefasste Begriff der Spiritualität bietet auch die Chance für alle Menschen, unabhängig von der Religionszugehörigkeit, offen zu sein, selbst für nicht religiöse Menschen, die sich aber mit existenziellen Sinnfragen beschäftigen. Gleichzeitig wird damit der Begriff derart erweitert, dass spirituelle Bedürfnisse kaum noch von psychosozialen Bedürfnissen zu unterscheiden sind. Die in der Literatur beschriebenen spirituellen Bedürfnisse lassen sich in vier miteinander verbundene Kerndimensionen differenzieren:

1. Verbundenheit (Liebe, Zugehörigkeit, Partner-Kommunikation etc.),

2. Friede (innerer Friede, Hoffnung, Ausgeglichenheit, Vergebung etc.),
3. Sinn/Bedeutung (Lebenssinn, Selbstverwirklichung etc.) und

4. Transzendenz (spirituelle Ressourcen, Beziehung zu Gott/dem Heiligen, Beten etc.) (Büssing et al. 2012).

\section{Raumdesign kann Spiritualität und Spiritual Care begünstigen}

Ist es möglich, gestalterisch Atmosphären zu erzeugen, die diese vier Kerndimensionen spiritueller Bedürfnisse unterstützen? Und wenn ja, wie? Wie kann man Spiritualität fördernde räumliche Elemente und Aspekte auch in zumeist sehr funktional gestaltete Gesundheitsbauten integrieren, um Atmosphären zu erzeugen, die gar eine Begegnung mit dem Transzendenten ermöglichen können und die uns transparent machen für Sinnfragen und das Wesentliche? Wie vermag Architektur in uns emotionale Texturen wie Verbundenheit, Friede, Präsenz und Transzendenz zu begünstigen?

Orte und Räume können durch ihre Atmosphäre zu Stille, Präsenz, Kontemplation, Friede und Selbstfindung einladen und damit Spiritualität fördern. Um solche Orte zu schaffen, gilt es, sich bewusst zu machen, welche gestalterischen Elemente und Charakteristika solche Atmosphären begünstigen. Wichtige Quellen der Inspiration sind:

- Theoretische und praktische Ansätze der „Healing Architecture“

- Erkenntnisse, wie Patienten ihre Umwelt erleben

- Gestaltungscharakteristika sakraler Architektur

\section{Atmosphäre der Verbundenheit und Liebe}

Auf der menschlichen Ebene ist für einen Patienten kaum etwas so wichtig und wohltuend wie die empathische Anteilnahme und Zuwendung von Ärzten, Pflegern und vielleicht Mitpatienten, die Gemeinschaft mit Angehörigen und Freunden. Besonders heilsam sind Verbundenheit und Liebe in ihren spirituellen Formen. Der übliche Krankenhausalltag lädt zu solch heilsamer Verbundenheit und Liebe leider kaum ein, beginnend bei den ungünstigen räumlichen Bedingungen. Ein drastisches Beispiel dafür inspirierte als Reaktion eine berühmt gewordene frühe Initiative zu „Healing Architecture“: Die 13jährige Maria Fareri lag und starb 1995 auf der Kinderstation der Universitätsklinik Westchester Medical Center "WMC" (Valhalla bei New York). Ihre Eltern Brenda und John Fareri waren entsetzt über das Ambiente: Krankenzimmer und 
Flure waren mit Utensilien vollgestellt, beengt und überfüllt. Es gab kaum Platz für Besucher, und Privatheit war in dem allgegenwärtigen Lärm und Durcheinander unmöglich. Nach diesem traumatischen Erlebnis starteten Brenda und John Fareri eine Initiative zum Neubau einer separaten "familienzentrierten" Kinderklinik für das WMC (über eigene Mittel und Spenden). Leitideen für Errichtung, Einrichtung und Betrieb der „Maria Fareri Kinderklinik“ waren:

- Kinder sollen so normal wie möglich leben: in sozialer Geborgenheit lernen und sich verwirklichen können.

- Eltern und Besucher sind willkommen und Partner im Heilungsprozess.

- Schöne, zur Erholung, Gemeinschaft, Aktivität und Kreativität anregende Umgebung soll wesentlich zur Genesung beitragen.

Zur Verwirklichung dieser Ziele trug die räumliche Gestaltung bei:

- Insgesamt gibt es fünfmal mehr Platz als auf der alten Kinderstation.

- Statt unwirtlicher Flure gibt es einladende allgemein zugängliche Bereiche, wo Kinder und Besucher sich gern aufhalten.

- Innen- und Außenbereiche sind mit Blick auf Kinder und Familien gestaltet.

- Die Kinder haben große, ruhige, helle Einzelzimmer mit Fenster, Bad, Tisch, Telefon, TV, Ausziehsofa, auf denen Besucher auch die Nacht verbringen können.

- Es gibt spezielle Gemeinschaftsareale für diverse Beschäftigungen für jeweils definierte Altersklassen. So ist „Schifffahrt“ ein nautisch gestalteter Spielbereich um ein "Schiff". "Theater" ist ein Kunststudio mit kreativen Programmen usw.

- Besucher, die länger bleiben, haben Raum und Gelegenheit zu arbeiten, zu duschen, Wäsche zu waschen, zu kochen...

- Langzeit-Besuchern steht ein eigenes Haus mit Appartements zur Verfügung.

Solche Errungenschaften einer heilsamen und heilenden Architektur schaffen somit basale Voraussetzungen, dass Patienten, Mitarbeiter, Angehörige und Besucher sich in der schwierigen Umgebung Krankenhaus willkommen und wohl fühlen, dass Verbundenheit und Liebe sich besser entfalten können. Besonders in der letzten Phase des Sterbens ist die kontinuierliche Anwesenheit, das Dasein der Angehörigen für den Patienten hilfreich.

\section{Atmosphäre des Friedens und der Ruhe}

Ein spezieller Genius loci oder eine besonders schöne Lage in der Natur können wesentlich sein, um einen besonderen Ort zu schaffen. Zur spirituellen Dimension gehört das „intensive Erleben der Schönheit bzw. der Heiligkeit der Natur" (Grom 2011: 15). Hierzu gehören lichte und helle Zimmer, die einen weiten Blick in die Natur ermöglichen, wie auch die Möglichkeit in der Naturumgebung spazieren gehen zu können. Ganz wesentlich hierbei ist der schöne Ausblick in die Weite. Diese Weite nehmen wir leiblich wahr. Sie beeinflusst deutlich unsere Stimmung. Schon 1886 beschrieb der Kunsthistoriker Heinrich Wölfflin die Wechselwirkung von leiblicher Wahrnehmung und Stimmung. In großzügigen weiten Hallen können wir „tief und voll“ atmen, ,als wäre unsere Brust so weit wie die Hallen“, bemerkte Wölfflin (1886). Ebenso reagieren wir entspannt auf weite, schöne Ausblicke. Der Zusammenhang zwischen Atmen und Gesundheit ist heute längst erwiesen und gilt nicht mehr nur als fernöstliche Meditationsweisheit wie noch in den 70er Jahren. Ein Jahrhundert nach Wölfflin leiteten die Philosophen Hermann Schmitz (1980, 2003) und Gernot Böhme (1995) mit ihrer Leib-Phänomenologie die Renaissance einer stärkeren Beachtung des Leiblichen und Affektiven ein.

Ein weiter Ausblick auf Natur fördert die Erholung und der Stress kann schneller abgebaut werden (Hartig et al. 2003). Eine der ersten Studien zur positiven Auswirkung von Natur war die 1984 im Wissenschaftsmagazin Science veröffentliche Studie des Architekturprofessors Rogers Ulrich (1984), die zum Wegbereiter für das Konzept der Healing Architecture wurde. Er verglich zwei Gruppen von Patienten, die im Krankenhaus nach identischen Operationen (Gallenblasen-OP) durch ihre Zimmerfenster entweder auf einen Park mit Bäumen oder auf die Betonmauer des Nachbargebäudes sehen konnten. Patienten, die auf den Park sehen konnten, benötigten deutlich weniger Schmerzmittel, litten seltener an Depressionen und konnten im Schnitt einen Tag früher nach Hause entlassen werden als die Patienten der Vergleichsgruppe. Natur und Licht bewirken Studien zufolge auch, dass bei Patienten, die zur Traurigkeit und Depression neigen, sich die Stimmung aufhellt und die Depressionsrate sinkt" (Cohen-Cline et al. 2015).

Eine neuere Studie von Vollmer/Koppen (2018: 220) belegt, dass schwerstkranke Menschen besonders empfindlich auf Geruch, Privatheit und Weitsicht reagieren. Patienten empfinden ihre Intimsphäre tendenziell als nicht mehr ausreichend privat, als eingeengt und von unangenehmen Gerüchen durchdrungen. Der Ausblick in die Weite der Natur wird zur Notwendigkeit, um der Enge des 
erkrankten Körpers zu entweichen. Die Weite der Natur wird inkorporiert, um aus der Enge des schwer erkrankten Körpers auszubrechen.

Zu häufig sind Ausblick in die Natur oder auch Spazierengehen im Grünen in Krankenhäusern nicht möglich. Bei der Planung sollte schon die Lage des Ortes eine Rolle spielen. Wenn umgebende Natur und Grün nicht ausreichend vorhanden sind, sollte dies landschaftsplanerisch korrigiert werden. Heute ist es möglich, auch auf kleinen Arealen grüne Oasen wie begrünte Innenhöfe, begrünte Dachterrassen oder vertikale Gärten zu erschaffen. Licht und Natur mit ihren Rhythmen vom Morgengrauen bis zur Abenddämmerung bieten nicht nur das Erleben angeschauter Schönheit, sondern auch einen Zugang zum inneren Frieden, zur Ruhe und Ausgeglichenheit.

Ganz entscheidend ist aber auch die Vermeidung von Stress durch schlechte Gestaltung, da die Wirkung der Umgebung bei kranken Menschen intensiver als bei gesunden wahrgenommen wird. Je weniger wir uns mit unserem Körper wohlfühlen, umso empfindlicher und verletzlicher reagieren wir auf unangenehme sensorische Reize wie laute Geräusche, sehr helles oder flackerndes Licht, schlechte Gerüche, leuchtende Farben, hohe Dichte. Neben einer Überstimulierung durch Reize kann auch monotone und lieblose Gestaltung Stress erzeugen. Wie sehr die ästhetische Gestaltung zum Wohlbefinden oder auch Stresserleben beitragen kann, belegten Gary W. Evans und Janetta Mitchell Mc Coy von der Cornell University in Ithaca (USA) 1998 mit ihrer Publikation „When buildings don’t work“ (Evans, McCoy 1998). Die beiden Psychologen stellten fünf Dimensionen - die sogenannten BIG FIVE - zusammen, die eine entscheidende Rolle für das Wohlergehen der Menschen spielen:

- Stimulierung,

- Affordanz,

- Kohärenz,

- Kontrolle und

- Erholung.

Diese fünf Dimensionen wirken erheblich auf unser Befinden, auch weil wir ihre Aspekte und Bedingungen nicht nur visuell, sondern vieldimensional synästhetisch leiblich erleben.

Die BIG FIVE sind eine Checkliste für unser Befinden und unseren Stresslevel. Immer noch sind viele Gesundheitsbauten charakterisiert durch lange, abweisende Korridore (schlechte Affordanz), unwohnliche Räume, verwirrende Unübersichtlichkeit (schlechte Orientierung und Kohärenz) und störenden Lärm (Überstimulierung) und geringe interpersonelle Distanzen (Kontrollverlust). Die umliegenden Flächen sind oft vollständig asphaltiert, wir- ken unwirtlich und öde (keine Erholung). Eine solche Umgebung erzeugt Stress, schädigt nachweisbar Leib und Psyche und verzögert Heilungsprozesse. Dieser Stress kommt zusätzlich zu den vielen belastenden Momenten, mit denen chronisch und schwer Kranke konfrontiert sind, als da sind: schwierige Arzt-Patienten-Gespräche, anstrengende Untersuchungen, Wartezeiten, körperliches Unwohlsein, Existenzängste und Schmerzen. So kann ein Krankenhauszimmer mit Lärmbelastungen dazu führen, dass der Patient sich nicht nur besorgt, traurig oder hilflos fühlt, sondern dass seine Schlafqualität gestört wird und sich auch sein Blutdruck und seine Herzfrequenz sowie seine Muskelspannung erhöhen können (Stern 2011: 228). Studien zeigen, dass schon bei gesunden Menschen bereits ein chronischer Lärmpegel von über 65 Dezibel tagsüber das Risiko für Herz-Kreislauf-Krankheiten deutlich ansteigen lässt (Babisch 2004). Ist man erkrankt, steigt das Stresserleben. Umso wichtiger ist also die Gestaltung einer angenehmen Atmosphäre und Stimmung, die Genesung fördert.

\section{Atmosphäre für Sinnsuche und Präsenz}

Sinnsuche, Selbstakzeptanz und Selbstentfaltung werden gefördert von Räumen der Ruhe und Stille, da diese sich in eine stimmungsmäßige Ruhe und innere Stille übertragen. In Krankenzimmern, die von vielen Reizen überflutet sind, wie Hektik des Personals, lauten Geräuschen, unruhiger Gestaltung, schlechten Gerüchen und eventuell noch Straßenlärm, ist es schwerer möglich, sich auf spirituelle Stimmung und Gedanken einzulassen. Hochwertig gestaltete Räume, die eine reizmäßig reduzierte und warme Atmosphäre aufweisen, laden eher zu Selbstbesinnung und Ruhe ein. Eine eher einfache Gestaltung der Zimmer und das Fehlen des Überflüssigen können heilsam wirken, wenn sie auf der mönchischen Idee beruhen, den Geist befreien zu wollen auf dem Weg der geistigen Erleuchtung und Selbsterkenntnis. Eine reduzierte Gestaltung der Architektur und der Verzicht der Mönche auf privates Eigentum bedeuten jedoch nicht, dass Mönche in ärmlichen Räumen leben. So gibt es im Testament des Franziskus den wichtigen Unterschied zwischen einer ärmlichen Wohnung und der Heiligen Armut (Hasse 2009: 103). „Danach kann eine Wohnung ärmlich sein, wenn sie schlecht oder unzureichend ausgestattet ist; Ein Leben in heiliger Armut dagegen erfordert eine Art Ausstattung, die die Erfahrung der Gegenwart des Numinosen ermöglicht. Eine Wohnung, die nur ärmlich ist, mag vieles in schlechter Qualität bieten, aber keinen Rahmen für die Entstehung göttlicher Atmosphären. Klöster sind aber reine Räume der Atmo- 
sphären“ (Hasse 2009: 103). Das heißt: Die Gestaltung muss auch in der größten Reduktion und Abkehr von Bildlichkeit eine gestalterische Qualität aufweisen, um ein kontemplatives Innehalten zu ermöglichen. Ähnliches findet sich in der japanischen Tradition: Die Leere des Raumes, „gar die Erfahrung der In-Differenz“ der All-heit (die weder trennt noch unterscheidet), fördere „eine erhöhte Konzentration und kontemplative Aufmerksamkeit für das Wahrnehmbare“ (Haepke 2013: 143).

Einer der elementarsten Beiträge zur Atmosphäre ist die Lichtgestaltung. Licht erfüllt atmosphärisch den ganzen Raum. Es gibt viele emotionale Entsprechungen von Licht und Gefühlen. Wir erfreuen uns am belebenden heiteren Morgenlicht und am weichen warmen Abendlicht, während graue trübe Lichtverhältnisse eher unsere Stimmung drücken. Viele unsere Befindlichkeiten werden mit Lichtmetaphern beschrieben wie Strahlen, helle oder dunkle Stimmung. In Krankenhäusern erzeugt das starke, künstliche, grelle Licht eine kalte und sterile Atmosphäre. Warmes indirektes Licht, aber auch möglichst viel natürliches Licht tragen zum Wohlbefinden bei. Das natürliche Licht und die Dunkelheit regulieren die gegenläufigen Zyklen der Hormone Cortisol und Melatonin. Grob gesprochen unterstützt Cortisol energetisierende und stimulierende Prozesse, während wir unter dem Einfluss von Melatonin müde werden und der Organismus auf Sparflamme und Erholung schaltet. In vielen Krankenhäusern wird kühlweißes künstliches Licht gezielt eingesetzt, damit die Mitarbeiter nicht müde werden. Wer den ganzen Tag bis in den Abend hinein unter dem kalten Neon-Licht lebt, stört seinen eigenen natürlichen Rhythmus (Arvay 2018: 258). Von Natur aus ist der Mensch darauf gepolt, im Hellen wach und fit zu sein, nach einer Phase der Dämmerung setzt dann im Dunkeln die Müdigkeit ein. Wird der Biorhythmus gestört, kann es zu „Erschöpfung, gedrückter Stimmung und Depression“ führen (Arvay 2018: 258). Viele Menschen unterschätzen den negativen Einfluss, den künstliches Licht auf die biologische Uhr hat.

\section{Atmosphäre der Transzendenz}

Weitere Dimensionen von Spiritualität sind Achtsamkeit und andere Meditationserfahrungen. Hierzu braucht es Räume, die zur inneren Einkehr einladen. Eine reduzierte Gestaltung und Abkehr von Bildlichkeit bei einer hohen gestalterischen Qualität kann diese Atmosphäre des kontemplativen Innehaltens und der Präsenz erzeugen. Auch die Atmosphäre einer „heiligen Dämmerung“ kann dazu beitragen, das Äußere und Alltägliche abzulegen, so dass tiefe Ernsthaftigkeit, Selbstwahrnehmung und Konzentra- tion auf das Wesentliche die weiteren Gedanken bestimmen. Das gedämpfte Licht der „heiligen Dämmerung“ hat in kirchlichen Räumen etwas sehr Bergendes. Nicht Oberlichter tauchen die Säle in gleichmäßiges Licht, sondern geschosshohe, unsichtbar gehaltene Fensterflächen erzeugen eine wechselvolle Spannung aus Licht und Schatten, Transparenz und Geschlossenheit.

Die Absonderung des Mediationsraumes durch einen Schwellenbereich und verlangsamende Wegeführungen tragen ganz wesentlich zur sakralen Atmosphäre bei. Typisch für sakrale Inszenierungen ist, dass sie Wege-Konzepten unterliegen, die die Besucher „in Raumsequenzen sukzessiv vom Alltag weg-führen“ (Haepke 2013: 315). Im Sinne eines multi-sinnlichen Erlebens kann ein umhüllender Geruch, gedämpftes, von oben hereinfallendes Licht, Kerzenschein (Sehen), Ruhe (Akustik), besondere Materialität, die Raumhöhe (visuell-propriozeptive Synästhetik) sowie die szenische Ausrichtung, zur meditativen und sakralen Stimmung beitragen.

Ein sinnvoller und wichtiger Ansatz bei der Gestaltung von Gesundheitsbauten ist es, die Perspektive des Patienten einzunehmen. Früher stand nur die Technik im Vordergrund, nun wird auch auf die Bedürfnisse des Patienten eingegangen. Worauf schaut der Patient, wenn er im Bett liegt? Worauf ist der Blick des Patienten gerichtet, wenn er ein schwieriges Gespräch mit dem Arzt führt? Neuere Studien deuten zunehmend darauf hin, dass Kranke ihre Welt anders wahrnehmen als Gesunde. Beispielsweise zeigte Tanja Vollmer, dass Krebskranke auch den äußeren Raum als „erkrankt“ empfinden können. Krebspatienten in der Psychoonkologie berichten über eine veränderte Wahrnehmung seit der Krebsdiagnose: „Räume werden farbloser wahrgenommen als vor der Erkrankung, Einrichtungsgegenstände als bedrückend dunkel erlebt. Außenräume verlieren ihren Bekanntheitsgrad, Wohlfühlen und Orientieren sind auf einmal keine Selbstverständlichkeiten mehr“ (Vollmer \& Koppen 2010: 33, 34). Schon in der Gesprächstherapie mit Krebspatienten war der Psychologin Vollmer aufgefallen, „dass Kranke ihre Empfindungen und Situationen meist mit räumlichen Metaphern beschreiben. Viele sprachen von räumlicher Enge, von tiefen, schwarzen Löchern, in die sie gefallen seien“, von einem „asymmetrisch“ erlebten Körper oder einem Körper, der nicht mehr im Gleichgewicht sei (Vollmer \& Koppen 2010: 13). Auch erzählten die Patienten von „plötzlichen Verdunklungen und Verengungen ihrer Räume“ sowie vom Verlust von Ruhe- und Rückzugszonen innerhalb ihrer gebauten Umgebungen (Vollmer \& Koppen 2010: 116). Der Wohn- und Lebensraum, der eigentliche Schutzraum, wird nun schwarz, dunkel, tief, eng, erdrückend, „beängstigend, laut und überfüllt“" wahrgenom- 
men und wird zur Bedrohung (Vollmer \& Koppen 2010:116). Der Wunsch nach „Weite, Aufbruch, Licht, Aussicht, Stille und Entleerung“ erwacht (Vollmer \& Koppen 2010: 117). Es ist sehr eindrücklich, wie stark Krankheit und Verzweiflung mit der Orientierung verknüpft sind. Die Schilderungen der Krebspatienten sind nicht nur metaphorisch. Sie gleichen „einer echten Wahrnehmung“ (Vollmer \& Koppen 2010: 131). Es verwundert daher nicht, dass eine derart zum Negativen veränderte Raumwahrnehmung Patienten veranlasste, ihre Wohnräume neu zu gestalten - etwa mit neuen Farbanstrichen, baulichen Veränderungen, um mehr größeren Lichteinfall zu ermöglichen, bis hin zu kompletten Veränderungen durch Umzug (Vollmer \& Koppen 2010: 116). Tatsächlich korrespondiert die gestalterische Umsetzung von Weite, Natur, Aussicht Licht und Stille auch mit den Wünschen sterbenskranker Krebspatienten.

Architektur kann Rahmenbedingen für Spiritualität und Spiritual Care schaffen. Doch ist dies nur eine förderliche Voraussetzung. Es braucht auch eine innere Haltung der Patienten und eine geeignete Betreuung im Sinne von Spiritual Care.

\section{Die Bedeutung synästhetischer leiblicher Resonanzen für die Gestaltung}

Heilsame leibliche Empfindungen generieren sich durch eine Architektur, die es vermag, das Tempo zu drosseln, Ruhe zu erzeugen, eine Verbindung zur Natur herzustellen, gelichtete und großzügige, atmende Räume zu gestalten und auf das würdige Altern der Materialien zu achten. Mit einer heilsamen Architektur ist vor allem der Ansatz verbunden, eine möglichst stressarme und erholsame Umgebung für den Patienten zu schaffen. Spirituelle Anmutungen (in der Designpraxis bezeichnet man mit Anmutung die von einem Entwurf geweckten, oft unbewussten Erwartungen) können gezielt eingesetzt werden, um eine rituelle Distanz zum Alltag zu evozieren, welche Erbauung und Besinnung, Erhebung und Andacht provozieren. Die räumliche Gestaltung kann es den Patienten ermöglichen, inmitten der funktional und technisch kontrollierten Abläufe, die sie kontrollieren, für sich selber wieder ein Stück Lebensqualität und Selbstbestimmung zu gewinnen. Ästhetische Gestaltung ist elementar für unser Befinden und ist weniger eine künstlerisch, formalistische Angelegenheit. „Aisthesis meint die sinnlich-affektive Teilnahme an den Dingen“ (Böhme 1995: 51). Die gestaltete Umgebung bildet für den Menschen einen emotionalen Resonanz- raum, der das Individuum mit seinen Stimmungen und Befindlichkeiten in den Mittelpunkt rückt. Ästhetische Wahrnehmung wird von den leiblichen Resonanzen bestimmt und beeinflusst unser Wohlbefinden in der Umwelt oder erzeugt gar auch Stress. Durch das Wissen um die psychophysische Wechselwirkung wird es möglich zu verstehen, wie die Umgebung emotionale Reaktionen beeinflusst und wie Räume durch ihre Gestaltung zur Gesundheit, Entspannung, Entschleunigung, Spiritualität und Gemeinschaft beitragen können. Eine erlebbare Architektur, die das leiblich-räumliche Spüren auf eine vielschichtige, synästhetische Weise induziert und fördert, verstärkt das Gefühl des Daseins und des Involviertseins in der materiell-sozial-kulturellen Welt. Gerade im Umgang mit schwerstkranken Menschen können Gefühle wie innerer Friede, Hoffnung, Ausgeglichenheit, Vergebung, Lebenssinn, Selbstverwirklichung durch die Gestaltung unterstützt werden und die extremste Daseinskonfrontation mit dem Tode lindern.

Interessenkonflikt: Die Autorin bestätigt, dass kein Interessenkonflikt vorliegt.

\section{Literatur}

Arvay CG (2018) Biophilia in der Stadt. Wie wir die Heilkraft der Natur in unsere Städte bringen. München: Wilhelm Goldmann.

Babisch W (2004) Chronischer Lärm als Risikofaktor für den Myokardinfarkt. Umweltbundesamt Berlin.

Boothe B, Frick E (2017) Spiritual Care. Über das Leben und Sterben. Zürich: orell füssli.

Böhme G (1995) Atmosphäre. Essays zur neuen Ästhetik. Frankfurt am Main: Suhrkamp.

Büssing A, Janko A, Kopf A, Lux E, Frick E (2012) Zusammenhänge zwischen psychosozialen und spirituellen Bedürfnissen und Bewertung von Krankheit bei Patienten mit chronischen Erkrankungen. Spiritual Care 1:57-73.

Bruns F, Steinmann D, Micke O. (2007) Spiritualität in der Onkologie. Onkologie 13 (6):490-498.

Cohen-Cline H, Turkheimer E, Duncan GE (2015) Research report. Access to green space, physical activity and mental health. A twin study. Journal of Epidemiology and Community Health 69:523-529.

Evans GW, McCoy JM (1998) When buildings don't work. The role of architecture in human health. Journal of Environmental Psychology 18:85-94.

Frick E (2007) Sich heilen lassen. Eine spirituelle und psychoanalytische Reflexion. Würzburg: echter.

Grom B (2011) Spiritualität - die Karriere eines Begriffs: Eine religionspsychologische Perspektive. In: Frick E, Roser T (Hg.) Spiritualität und Medizin. Gemeinsame Sorge für den kranken Menschen. Stuttgart: Kohlhammer. 12-17.

Haepke N (2013) Sakrale Inszenierungen in der zeitgenössischen Architektur. Bielefeld: transcript. 
Hasse J (2009) Unbedachtes Wohnen. Bielefeld: transcript.

Hartig T, Evans GW, Jamner LD, Davis DD, Gärling T (2003) Tracking restoration in natural and urban field settings. Journal of Environmental Psychology 23/2:109-123.

Roser T (2009) Spiritual Care - neuere Ansätze seelsorglichen Handelns. In: Körtner U, Müller S, Kletecka-Pulker M (Hg.) Spiritualität, Religion und Kultur am Krankenbett (Schriftenreihe Ethik und Recht in der Medizin, Bd 3). Wien: Springer.

Sternberg EM (2011) Heilende Räume. Die Wirkung äußerer Einflüsse auf das innere Wohlbefinden. Amerang: Crotona.

Siepmann H (2017) Interview am 1.11.2017.

Schmitz H (1980) Neue Phänomenologie, Bonn: Bouvier.

Schmitz H (2003) Was ist Neue Phänomenologie? Rostock: Koch. Ulrich RS (1984) View through a window may influence recovery from surgery. Science 224/4647:420-421.

Vollmer TC, Koppen G (2010) Die Erkrankung des Raumes.

Raumwahrnehmung im Zustand körperlicher Versehrtheit und deren Bedeutung für die Architektur. München: Herbert Utz.
Vollmer TC, Koppen G (2018) Architekturwahrnehmung und Stresserleben schwerst- und chronisch Kranker. In: Abel A, Rudolf B ( $\mathrm{Hg}$.) Architektur wahrnehmen, Bielefeld: transcript. 207-230.

Wölfflin H (1886) Prolegomena zu einer Psychologie der Architektur. München: Kgl. Hof \& UniversitätsBuchdruckerei von Dr. C. Wolf \& Sohn.

\section{Biografische Angaben}

\section{Katharina Brichetti}

PD Dr.- Ing. habil.; Privatdozentin für Bau- und Stadtgeschichte an der Technischen Universität Berlin. Seit 2011 forscht und lehrt sie zum Thema „Architektur und Gesundheit“. Buchpublikationen: Das Gedächtnis der Stadt, 2006; Die Paradoxie des postmodernen Historismus, 2009; Berlins neue Mitte, 2011; Synästhesie: LeibRaum-Architektur, 2014 mit Franz Mechsner; Heilsame Architektur, 2019 mit Franz Mechsner. 